\title{
Cross-cultural French adaptation and validation of the Impact On Family Scale (IOFS)
}

\author{
Raphaël Boudas ${ }^{1}$, Jérémie Jégu ${ }^{1,2}$, Bruno Grollemund ${ }^{3,4^{*}}$, Elvire Quentel ${ }^{3}$, Anne Danion-Grilliat ${ }^{4,5}$ \\ and Michel Velten ${ }^{1,2,6}$
}

\begin{abstract}
Background: The IOFS (Impact On Family Scale) questionnaire is a useful instrument to assess the impact of chronic childhood conditions on general family quality of life. As this instrument was not validated in French, we proposed to translate, adapt and validate the IOFS questionnaire for clinical and research use in French-speaking populations.
\end{abstract}

Findings: The sample studied comprised French-speaking parents with a child presenting a cleft lip or cleft lip and palate, aged 6 to 12 years and treated in the University Hospital of Strasbourg, France. The 15-item version of the IOFS was translated into French and then sent to the parents by post. The structure of the measure was studied using Exploratory Factor Analysis (EFA), internal consistency was assessed using Cronbach's alpha coefficient and test-retest reliability was studied by calculating the Intraclass Correlation Coefficient (ICC).

A total of 209 parents answered the questionnaire. Its acceptability was good, with 67.9\% of mothers and 59.9\% of fathers answering the questionnaire. EFA identified one main factor that explained $77 \%$ of the variance. Internal consistency was good, with a Cronbach's alpha of 0.93. Finally, the ICC values were 0.77 ( $95 \%$ confidence interval 0.66-0.85) and 0.87 (95\% confidence interval 0.80-0.92) for inter- and intra-observer reliability respectively.

Conclusions: The French version of the IOFS questionnaire exhibited very good psychometric properties. For practitioners, this instrument will facilitate the assessment of the impact of chronic childhood conditions on quality of life among French-speaking families.

Keywords: Quality of life, Family, Child, Cleft lip, Cleft palate, Validation studies

\section{Background}

Quality of life measurement has become a major topic in pediatric medicine alongside the advances in medical care of children with chronic conditions [1]. In particular, chronic conditions have been reported to affect family functioning and family well-being [2]. The IOFS (Impact On Family Scale) questionnaire is an instrument measuring health and quality of life which enables quantification of the impact on the family of chronic childhood conditions. This instrument, initially developed by Stein et al. with 33 items and 4 dimensions [3] was progressively reduced to a 15 -item questionnaire

\footnotetext{
* Correspondence: bruno.grollemund@gmail.com

${ }^{3}$ Orthodontic Department, Faculty of dentistry, University of Strasbourg, 1

Place de I'Hôpital, Strasbourg, France

${ }^{4}$ Laboratoire éthique et pratiques médicales, IRIST EA 3424, Université de

Strasbourg, Rue de I'Université 7, Strasbourg, France

Full list of author information is available at the end of the article
}

with one main dimension representing general negative impact on the social and familial systems $[4,5]$. To date, validations of cross-cultural adaptations of the IOFS have been performed in different languages: Spanish, Italian, German, Portuguese and Turkish [6-10]. As this instrument was still not validated in French, we proposed to translate, adapt and validate the IOFS questionnaire for clinical and research use in French-speaking populations.

\section{Methods}

The guidelines proposed by Beaton et al. were used for the cross-cultural adaptation of the 15-item IOFS questionnaire [11]. Briefly, the IOFS was translated into French by two independent native French translators. A synthesis of the two questionnaires obtained was performed by an expert committee. Then the resulting French questionnaire was back-translated into English 
by two independent English-native translators and the two questionnaires obtained were reviewed by the expert committee. Finally, the French-translated questionnaire obtained was pre-tested on 30 people to ensure that the questionnaire was perfectly understandable and clear.

The population included during the validation process consisted of French-speaking parents with a child presenting a cleft lip, or a cleft lip and cleft palate, treated in the French University Hospital of Strasbourg. The children included were to be aged from 6 to 12 years and were not to have undergone surgery in the past 12 months.

The French-translated IOFS questionnaires were sent to the parents by post. These questionnaires were accompanied by a short questionnaire about the parents' socio-professional characteristics. Parents were asked to return completed questionnaires to the investigation center by post. Finally, a second IOFS questionnaire was sent 15 days after the first one for the purpose of interobserver reliability assessment. In addition, clinical data about the children (gender, age and type of cleft) were collected using the University Hospital of Strasbourg patient database.

Acceptability was assessed on the basis of the proportion of parents answering the first IOFS questionnaire. The total impact score was calculated by summing the results of all items, giving a total score ranging from 15 (no impact) to 60 (maximum impact). It should be noted that at this step of the analysis, we had to reverse the item values attributed (1 becoming 4, 2 becoming 3 and so on...) to obtain higher IOFS score values for greater impact. Median and inter-quartile ranges were used to describe IOFS total score distribution. Then the structure of measure was studied using an Exploratory Factor Analysis (EFA), a variable reduction technique which identifies the number of latent constructs and the underlying factor structure of a set of variables [12]. The internal consistency of the measure was assessed by calculating Cronbach's alpha coefficient. The children were considered as the statistical unit $(n=113)$ for EFA and internal consistency assessment. If both parents answered to the questionnaire, we restricted the analyses to the answers provided by the mother, as the answers from parent couples could not be considered independent. Finally, inter-observer reliability (between parents of the same child) and intra-observer reliability (between the two questionnaires completed within 15 days of each other) were assessed by calculating the Intraclass Correlation Coefficient (ICC). All analyses were performed using SAS version 9.2 (SAS Institute, Cary, North Carolina).

\section{Results}

A total of 327 questionnaires were sent by post to the parents of the children included. Among the parents contacted, 209 (63.9\%) completed and returned the
IOFS. Acceptability was slightly better among mothers compared to fathers, with response rates of $67.9 \%$ and $59.9 \%$ respectively. A minority of parents were divorced or single $(28.4 \%$ of the mothers and $22.6 \%$ of the fathers), the educational level was higher among mothers compared to fathers $(49.5 \%$ had a higher education diploma versus $32.6 \%$ ) and a majority of parents had an individual monthly income between 1500 and $3000 €(40.4 \%$ among mothers and $48.9 \%$ among fathers). Concerning the 113 children included, there was a majority of boys (65.5\%), the mean age was 8.5 years (SD 1.8) and most of them presented a unilateral cleft lip and cleft palate (52.2\%). The median score on the IOFS was 17 with an inter-quartile range of $15-24$.

EFA, which was not restricted at the outset for the number of factors, identified one main factor which explained $77 \%$ of the variance, followed by a second one which explained only $10 \%$ of the variance. The results of factor loadings obtained by restricting EFA to one factor are shown in Table 1. The items presenting the highest loadings were: "family gives up things" (IMPT), "see family and friends less" (IMPK), "fatigue is a problem" (IMPU) and "hard to find reliable person to care for child" (IMPI).

The assessment of internal consistency yielded a Cronbach's alpha of 0.93 .

Finally, ICC values were $0.77 \quad(95 \%$ confidence interval $0.66-0.85)$ and 0.87 (95\% confidence interval 0.80-0.92) for inter- and intra-observer reliability respectively.

Table 1 Factor loadings of fifteen Impact on Family Scale items; one-factor principal component solution $(n=113)$

\begin{tabular}{llr}
\hline Item label & Text (abbreviated) & Item loading \\
\hline IMPU & Fatigue is a problem & 0.81 \\
\hline IMPK & See family and friends less & 0.83 \\
\hline IMPJ & Need to change plans at last minute & 0.76 \\
\hline IMPH & Little desire to go out & 0.71 \\
\hline IMPR & No time for other family members & 0.75 \\
\hline IMPV & Live from day to day & 0.67 \\
\hline IMPI & Hard to find reliable person to care for child & 0.81 \\
\hline IMPT & Family gives up things & 0.88 \\
\hline IMPW & Nobody understands the burden & 0.73 \\
\hline IMPF & Can't travel out of city & 0.70 \\
\hline IMPAA & Live on roller coaster & 0.74 \\
\hline IMPG & People treat us special & 0.73 \\
\hline IMPX & Travel to hospital is a strain & 0.58 \\
\hline IMPO & Think about not having more children & 0.49 \\
\hline IMPM & Wonder whether to treat child "specially" & 0.65 \\
\hline
\end{tabular}




\section{Discussion}

The French version of the IOFS questionnaire showed very good psychometric properties. During the validation process, EFA identified one main factor explaining most of the variability, which is consistent with the results previously published by Stein et al. and Williams et al. for the 15-item version of the IOFS [4,5]. The results of factor loadings obtained in our study differed slightly from those reported in these articles. This could be explained by the range of physical disorders among children included in the Pediatric Ambulatory Care Treatment Study (PACTS), the Sharing The Experience of Parenting study (STEP), the Family Advocacy and Coordination Effort study (FACE) and the Intervention for Siblings study (ISEE), compared to the homogenous population of children presenting a cleft lip, or a cleft lip and a cleft palate considered in our study. However, it should be noted that the items "See family and friends less" (IMPK) and "Fatigue is a problem" (IMPU) nevertheless presented among the highest loadings in our study, consistent with the results obtained with the PACTS, STEP, FACE and ISEE datasets. The internal consistency of the French-adapted IOFS was very good (Cronbach's alpha was 0.93), which agrees with the estimates of $0.89,0.88$ and 0.83 in PACTS, STEP and FACE validation studies respectively [4]. Finally, testretest reliability was good, with an ICC of 0.87 , which can be compared to the 0.94 ICC estimate for the general impact dimension in the Turkish version of the IOFS [10].

In conclusion, the French IOFS questionnaire is a reliable instrument to assess the impact of chronic childhood conditions on quality of life among Frenchspeaking families. This instrument will be of immediate use in an ongoing prospective, multidisciplinary and multi-centre French study aiming to explore the perceptions and feelings of parents in the year following the birth of a child with cleft lip and palate, and to analyse parent-child relationships [13].

\section{Abbreviations}

IOFS: Impact on family scale; ICC: Intraclass correlation coefficient; EFA: Exploratory factor analysis; PACTS: Pediatric ambulatory care treatment study; STEP: Sharing the experience of parenting; FACE: Family advocacy and coordination effort; ISEE: Intervention for siblings.

\section{Competing interests}

The authors declare that they have no competing interests.

\section{Authors' contributions}

RB made substantial contributions to the conception and the design of the cross-cultural adaptation and validation of the questionnaire and wrote the first draft of the article. JJ performed the statistical analyses, data interpretation and revised the manuscript. BG coordinated the study and the data collection and revised the manuscript. EQ participated in all stages of this study, and was particularly involved throughout the data collection process. ADG revised the manuscript. MV participated in the study management, statistical analysis and revised the manuscript. All authors have read and approved the final manuscript.

\section{Acknowledgements}

We would like to thank Dr Stein R.E.K., Riessman C.K. and Dr Jessop D. for their permission to use and to perform a translation of the original version of the IOFS.

The Impact on Family Scale (@1978 by R. E. K. Stein, M.D., and Catherine Kohler Riessman, Ph.D.) is available for use with permission from the first author: Ruth E. K. Stein, M.D., Department of Pediatrics, Albert Einstein College of Medicine/Children's Hospital at Montefiore Medical Center, 111 East 210 Street, Bronx, NY 10467; e-mail: rstein@aecom.yu.edu. The French-translated version of the IOFS is also available for use from Ruth E. K. Stein, M.D.

\section{Author details}

'Department of Epidemiology and Public Health, EA 3430, Faculty of Medicine, University of Strasbourg, 4 rue Kirschleger, Strasbourg 67000, France. ${ }^{2}$ Department of Public Health, University Hospital of Strasbourg, 1 Place de l'Hôpital, Strasbourg 67000, France. ${ }^{3}$ Orthodontic Department, Faculty of dentistry, University of Strasbourg, 1 Place de l'Hôpital, Strasbourg, France. ${ }^{4}$ Laboratoire éthique et pratiques médicales, IRIST EA 3424, Université de Strasbourg, Rue de I'Université 7, Strasbourg, France. ${ }^{5}$ Service Psychothérapique pour Enfants et Adolescents, Hôpitaux Civils, Hôpitaux Universitaires de Strasbourg, Place de l'Hôpital 1, Strasbourg 67000, France. ${ }^{6}$ Department of Epidemiology and Biostatistics, Paul Strauss Comprehensive Cancer Center, 3 rue de la porte de l'hôpital, Strasbourg 67000, France.

Received: 12 November 2012 Accepted: 11 April 2013 Published: 23 April 2013

\section{References}

1. Eiser $C$, Morse R: The measurement of quality of life in children: past and future perspectives. J Dev Behav Pediatr 2001, 22:248-256.

2. Stein REK: Caring for Children with Chronic Illness. New York: Springer; 1989.

3. Stein REK, Riessman CK: The development of an impact-on-family scale: preliminary findings. Med Care 1980, 18:465-472.

4. Stein REK, Jessop DJ: The impact on family scale revisited: further psychometric data. J Dev Behav Pediatr 2003, 24:9-16.

5. Williams AR, Piamjariyakul U, Williams PD, Bruggeman SK, Cabanela RL: Validity of the revised Impact on Family (IOF) scale. J Pediatr 2006, 149:257-261.

6. Stein RE, Jessop DJ: Measuring health variables among Hispanic and non-Hispanic children with chronic conditions. Public Health Rep 1989, 104:377-384.

7. Kolk AM, Schipper JL, Hanewald GJFP, Casari EF, Fantino AG: The Impacton-Family Scale: a test of invariance across culture. J Pediatr Psychol 2000, 25:323-329.

8. Ravens-Sieberer U, Morfeld M, Stein RE, Jessop DJ, Bullinger M, Thyen U: [The testing and validation of the German version of the impact on family scale in families with children with disabilities]. Psychother Psychosom Med Psychol 2001, 51:384-393.

9. Barbosa T de S, Gavião MBD: Evaluation of the Family Impact Scale for use in Brazil. J Appl Oral Sci 2009, 17:397-403.

10. Bek N, Simsek IE, Erel S, Yakut Y, Uygur F: Turkish version of impact on family scale: a study of reliability and validity. Health Qual Life Outcomes 2009, 7:4.

11. Beaton DE, Bombardier C, Guillemin F, Ferraz MB: Guidelines for the process of cross-cultural adaptation of self-report measures. Spine 2000, 25:3186-3191.

12. Suhr DD: Principal Component Analysis versus Exploratory Factor Analysis, SUGI 30 Proceedings. Philadelphia, Pennsylvania: Paper; 2005:203-230.

13. Grollemund B, Guedeney A, Vazquez M-P, Picard A, Soupre V, Pellerin P, Simon E, Velten M, Dissaux C, Kauffmann I, Bruant-Rodier C, Danion-Grilliat A: Relational development in children with cleft lip and palate: influence of the waiting period prior to the first surgical intervention and parental psychological perceptions of the abnormality. BMC Pediatr 2012, 12:65.

\section{doi:10.1186/1477-7525-11-67}

Cite this article as: Boudas et al: Cross-cultural French adaptation and validation of the Impact On Family Scale (IOFS). Health and Quality of Life Outcomes 2013 11:67. 